\title{
Das Dilemma der klinischen Register in der pädiatrischen Onkologie und Hämatologie
}

\author{
Pitfalls of Clinical Registries in Pediatric Oncology and Hematology
}

U. Göbel

O. Witt

Institut

Heinrich-Heine-Universität

Düsseldorf

\section{Bibliografie}

DOI $10.1055 / \mathrm{s}-2008-1073159$

Klin Pädiatr 2008; 220: 129-133

(c) Georg Thieme Verlag KG

Stuttgart · New York

ISSN 0300-8630

Korrespondenzadresse

Prof. em. Dr. U. Göbel

Heinrich-Heine-Universität

Düsseldorf

Moorenstr. 5

40225 Düsseldorf

Goebel@med.uni-duesseldorf. de
Bösartige Erkrankungen bei Kindern und Jugendlichen sind selten, da jährlich nur etwa 14 von 100000 Kindern und Jugendlichen neu erkranken [29]. Die Diagnosen und das biologische Verhalten unterscheiden sich gegenüber Erwachsenen erheblich, sodass die bei Erwachsenen gewonnenen Erkenntnisse nur in stark eingeschränktem Umfang auf Kinder übertragen werden können. Selbst bei Spezialisierung auf die malignen Erkrankungen des Kindes- und Jugendalters kann ein Arzt pro Jahr nur eine begrenzte Zahl von Patienten betreuen, die zudem unterschiedliche Diagnosen haben. Da das Alter und das Ausbreitungsstadium zum Diagnosezeitpunkt zudem wesentlichen Einfluss auf den Krankheitsverlauf haben, ist es heute einer Einzelperson nicht mehr möglich, eigenständig auf Evidenz basierende Erkenntnisse zur Tumorbiologie zusammenzutragen. Insofern ist es folgerichtig gewesen, nicht nur kooperativ und interdisziplinär Protokolle für die Diagnostik und Therapie zu entwickeln, sondern die Einzelergebnisse zusammenzuführen und gemeinsam kritisch auszuwerten. Dies hat sich die Gesellschaft für Pädiatrische Onkologie und Hämatologie (GPOH) als wissenschaftliche Fachgesellschaft zu einer ihrer Hauptaufgaben gemacht und ist deshalb auch feste Kooperationen mit Wissenschaftlern anderer Disziplinen eingegangen.

\section{Referenzbegutachtung bei seltenen Tumoren \\ $\nabla$}

Um bei den bösartigen Tumoren auf der Grundlage von einheitlich erstellten histologischen Diagnosen behandeln zu können, ist 1977 das Kieler Kindertumorregister gegründet worden [20]. Durch die enge Zusammenarbeit mit den behandelnden Ärzten und die Rückkoppelung der Behandlungsdaten sind neue Prognosefaktoren erkannt worden, die dann in nachfolgenden Therapieoptimierungsprotokollen zur Stratifikation der Therapie geführt haben. Neben die Referenzbegutachtung tritt die Erforschung der Tumorbiologie aus pathologisch-anatomischer Sicht unter Therapiebedingungen. Durch die Kombination von Immunhistologie und molekularbiologischen
Untersuchungen sind nicht nur Gemeinsamkeiten, sondern auch wichtige Unterschiede in der Ätiopathogenese der Tumoren des Kindesalters im Vergleich zu den Tumoren des Erwachsenenalters gefunden worden. Beispielhaft ist dies in dieser Zeitschrift erst kürzlich für die Teratome dargelegt worden; diese nach histologischen Kriterien gutartigen Tumoren sind während der zurückliegenden Jahrzehnte hinsichtlich ihrer Dignität aufgrund ihres biologischen Verhaltens sehr unterschiedlich beurteilt worden $[5,16,21]$. Die immunhistologische Referenzbegutachtung und die molekularbiologischen $\mathrm{Zu}$ satzuntersuchungen haben eine wissenschaftlichen Erklärung der unterschiedlichen Krankheitsverläufe und widersprüchlichen Beurteilungen geliefert.

\section{Risikostratifikation und individualisierte Therapie $\nabla$}

Weit komplexer gestaltet sich die Situation beim Neuroblastom, für das es wegen des Häufigkeitsgipfels in den ersten Lebensjahren keine Vergleichsmöglichkeiten mit dem Erwachsenenalter gibt. Schon frühzeitig sind trotz eindeutiger Zeichen der Malignität im histologischen Präparat Patientengruppen mit sehr guter, deutlich eingeschränkter und sehr schlechter Prognose nach gleichartiger Therapie erkannt worden [4]. Folglich wurde die Therapie für neu diagnostizierte Patienten nach der Risikozugehörigkeit stratifiziert, was für viele Patienten eine deutliche Reduktion der Therapie und für andere eine erhebliche Intensivierung zur Folge hatte [3]. Im Rahmen des bundesweiten Massenscreenings bei 1-jährigen Kindern zur Früherkennung des Neuroblastoms ist eine größere Zahl von Patienten identifiziert worden, die nach menschlichem Ermessen nicht symptomatisch geworden wäre, während sich die Zahl der Patienten mit dem ungünstigen Stadium IV nicht hat reduzieren lassen [39]. Diesen Kindern mit prognostisch besonders günstig einzustufendem Neuroblastom stehen andere gegenüber, bei denen trotz unauffälligem Screeningbefund wenig später eine unheilbare Tumorerkrankung diagnostiziert wurde. Die Untersuchung des Tumormaterials dieser Kinder 
mittels molekularbiologischer Verfahren hat zu einer Präzisierung der Risikoeinschätzung geführt. Heute können Kinder mit guter Prognose trotz eines initial fortgeschrittenen Tumors mit großer Zuverlässigkeit identifiziert werden, sodass selbst bei inkompletter Tumorresektion auf die früher als notwendig angesehene Chemotherapie oft verzichtet werden kann. Fischer et al. (siehe S. 137-146) geben hierzu in einer aktualisierten Übersicht den jetzigen Wissensstand der molekularbiologischen Diagnostik wieder [14].

In besonderen Notsituationen wird dennoch eine Chemotherapie notwendig sein, weil eine „watch-and-wait-stategy“ nicht ausreicht. Beispielhaft berichten Walter et al. (siehe S. 175-177) von einem Neugeborenen mit Lähmungserscheinungen als Folge eines sanduhrförmig in den Spinalkanal gewachsenen und klinisch diagnostizierten Neuroblastoms [43]. Da bei Kindern mit Neuroblastom bis zum Alter von 1 Jahr mit einer spontanen Rückbildung des Tumors gerechnet werden darf, werden diese Patienten heute einer „watch-and-wait-strategy“ zugeführt [22]. Als Einzelfallentscheidung ist bei diesem Neugeborenen aber aus klinischer Dringlichkeit notfallmäßig eine Chemotherapie mit offensichtlich gleichgünstigem Effekt verabreicht worden, wie aufgrund der Erfahrungen bei älteren Kindern durch eine Laminektomie zu erwarten gewesen wäre. Diese Operation ist bei dem Neugeborenen wegen der erforderlichen Ausdehnung als sehr eingreifend und der zu erwartenden erheblichen Spätfolgen als zu belastend eingestuft worden. Wegen der Seltenheit dieses Ereignisses ist die sorgfältige Dokumentation dieses Kindes in dem Kinderkrebsregister besonders wichtig, um dann bei Registrierung ähnlicher Patienten im Rahmen einer Spätfolgenermittlung zukünftig mehr Entscheidungssicherheit zu haben [26]. Die Regularien für derartige Spätfolgenerhebungen sind kürzlich durch die GPOH und das Kinderkrebsregister festgelegt worden, um einerseits methodisch einwandfreie Datenerhebungen durchzuführen und andererseits die ehemaligen Patienten durch zu häufige Anfragen nicht über Gebühr zu belasten [8].

\section{Zytogenetik und Immunphänotypisierung bei Leukämien \\ $\nabla$}

Auch für die einheitliche Diagnostik der Leukämien sind Referenzlabore und Register frühzeitig etabliert worden, die mittels Zytogenetik und Immunphänotypisierung sowie molekularbiologischer Techniken in Erweiterung der mikroskopischen Diagnostik prognostische Subgruppen bilden [18,34]. Durch die langjährige Tätigkeit dieser Register in enger Zusammenarbeit mit den behandelnden Kliniken und zuständigen Studienleitungen hat sich die Therapie für an Leukämie erkrankte Kinder gleichfalls hinsichtlich der Therapieintensität, der Therapiedauer und der Indikation für eine hämatopoetische Stammzelltransplantation geändert. Als konkretes Beispiel der interdisziplinären Kooperation dieser verschiedenen Register ist die Neubewertung des Alters als Risikofaktor bei der akuten lymphoblastischen Leukämie des Kindes- und Jugendalters zu nennen. Weltweit ist ein Alter über 10 Jahre als prognostisch ungünstig angesehen worden, woraus sich die Indikation für eine intensivere Therapie ableitet. Durch die Registerarbeit ist aber gezeigt worden, dass das Alter per se kein Risikofaktor ist, sondern dass mit zunehmendem Alter die unabhängig vom Alter identifizierten ungünstigen Prognosefaktoren häufiger und miteinander kombiniert auftreten [36].

\section{Epidemiologie und epidemiologische Forschung} $\nabla$

Einen inhaltlich ganz anderen Aufgabenbereich als diese Register, die sich von der Referenzbegutachtung ableiten, hat das epidemiologische Kinderkrebsregister, das 1980 an dem Institut für Medizinische Biometrie, Epidemiologie und Informatik in Mainz gegründet worden ist [18,34]. Die auf die Wohnbevölkerung bezogene Häufigkeitsermittlung für bösartige Erkrankungen ist aus sehr unterschiedlichen Gründen wichtig für die Gesundheitspflege, wie drei Beispiele belegen:

- Im Verbund mit den epidemiologischen Registern anderer Länder ist festgestellt worden, dass Krebserkrankungen bei Kindern während der zurückliegenden 20 Jahre langsam, aber stetig zugenommen haben [31].

- Die epidemiologische Ursachenforschung durch Befragungen im Rahmen von groß angelegten Fall-Kontroll-Studien stellt eine Hauptaufgabe dar. So sind aufgrund theoretischer Überlegungen angenommene oder aufgrund von Einzelbeobachtungen vermutete Ursachen für die Entstehung von bösartigen Erkrankungen im Kindes- und Jugendalter als unwahrscheinlich oder nur marginal beitragend erkannt worden $[27,30,35]$.

- Planungen zur Zahl und Größe kinderonkologischer Behandlungseinrichtungen durch den Bundesausschuss Krankenhaus erhalten durch die ständig aktualisierten Daten des Kinderkrebsregisters eine solide und jederzeit objektivierbare Grundlage [2].

Zusätzlich zu diesen originären Tätigkeiten hat das Kinderkrebsregister auch Aufgaben im Netzwerk der Patientenbehandlung übernommen. So übt es unter anderem die Brückenfunktion zwischen den Kliniken und den verschiedenen interdisziplinären Therapieoptimierungsprotokolle aus und gewährleistet auf diese Weise die schnelle und zuverlässige Zuordnung der neu erkrankten und gemeldeten Kinder und Jugendlichen an die entsprechende Studienleitung. Weiterhin übernimmt es die Langzeitnachsorge für die ehemaligen Patienten im Erwachsenenalter speziell auch unter dem Blickwinkel von malignen Zweiterkrankungen [26].

\section{Aufbau und Funktion von klinischen Registern \\ $\nabla$}

Von dem epidemiologischen Kinderkrebsregister sind die auf bestimmte Diagnosen ausgerichteten klinischen Register zu unterscheiden. Die relative Seltenheit der bösartigen Erkrankungen bei Kindern und Jugendlichen sowie die veränderte Tumorbiologie unter Therapiebedingungen haben dazu geführt, dass die Daten der im Rahmen der Therapieoptimierungsprotokolle prospektiv erfassten Patienten nach dem Ende der Studie nicht vernichtet, sondern in klinische Register für bestimmte Diagnosegruppen überführt werden. So ist bei jeder der etwa 25 Studienleitungen auch ein klinisches Tumorregister angesiedelt, in dem die krankheitsbezogenen Daten unter Berücksichtigung von Geschlecht, Alter, Stadium, Lokalisation, Risikofaktoren und Therapiemaßnahmen sowie Therapieergebnissen sorgfältig dokumentiert sind und regelmäßig aufdatiert werden [12]. Aufgrund der langfristig angelegten individuellen Nachsorgeuntersuchungen und der kontinuierlichen Zusammenführung der relevanten Daten in dem jeweiligen Register lassen sich das Risiko für späte Rezidive genauer definieren, die in Frage kommenden Rezidivorte präziser identifizieren und konsekutiv die Tumornachsorge auf eine zunehmend durch Evidenz gesicherte Grund- 
lage überführen. Hierdurch werden unnötige Untersuchungen vermieden und sowohl die ehemaligen Patienten als auch die Ärzte als Leistungserbringer sowie die Krankenkassen als Leistungsträger gleichermaßen entlastet. Auch können seltene Spätfolgen schneller erkannt und leichter von zufälligen Komorbiditäten unterschieden werden. Für den einzelnen Patienten kann somit die Erfassung in einem klinischen Register die frühzeitigere Diagnose einer seltenen Spätfolge und damit potenziell erfolgreichere Rehabilitation bedeuten. Für die Gesamtheit zukünftiger Patienten wird bei wiederholtem Auftreten bestimmter Spätfolgen aber eine geänderte Therapie resultieren. Die Verminderung oder vollständige Vermeidung von Spätfolgen im Sinne der primären Rehabilitation ist genauso stetes Anliegen der konsekutiven Therapieoptimierungsprotokolle wie die Verhinderung von Rückfällen oder die Reduzierung der akuten Toxizität. Deshalb werden in jedem Nachfolgeprotokoll für eine bestimmte Erkrankung alle Änderungen in der Therapie genau beschrieben und mit Hilfe der eigenen Registerdaten und der aktuellen Fachliteratur begründet.

Zur Unterstützung dieser Aufgaben sind Register zur prospektiven Erfassung von Spätfolgen und zur Beurteilung sowie Dokumentation der subjektiven gesundheitsbezogenen Lebensqualität eingerichtet worden [1,9]. Ergänzend kommt das Register für Kinder und Jugendliche hinzu, die im Rahmen ihrer onkologischen Therapie eine Bestrahlung erhalten haben [7].

Durch den Vergleich der Patientendaten, die in verschiedenen Protokollen mit kumulativ gleichen Zytostatika- oder Bestrahlungsdosen behandelt worden sind, gelingt es, die Einflüsse unterschiedlicher Applikationsweisen oder von Interaktionen zu erkennen. Da die weit überwiegende Mehrzahl der Patienten während der Strahlentherapie noch nicht ausgewachsen ist, kommt der Therapieplanung unter dem Aspekt der weitestgehend möglichen Schonung von gesundem Gewebe - insbesondere der Wachstumszonen - eine besondere Bedeutung zu. Deshalb ist auch die Erhebung zur Ausstattung und Inanspruchnahme von radiologischen Behandlungseinrichtungen unter kinderonkologischen Aspekten (siehe Seiten 178-182) ein wichtiger Schritt zur Qualitätssicherung und Therapieoptimierung [6]. Zudem führt der Vergleich zwischen ärztlich dokumentiertem Behandlungsergebnis und der subjektiv empfundenen Lebensqualität gelegentlich in der Einzelsituation zu divergierenden Feststellungen [10], was es im Rahmen zukünftiger Therapieplanungen zu berücksichtigen gilt. Akut wird die subjektive Lebensqualität der Patienten am stärksten durch die chemotherapieinduzierte Übelkeit und das Erbrechen beeinträchtigt, sodass medikamentöse und nichtmedikamentöse Begleittherapien mit zunehmendem Erfolg eingesetzt werden. Gottschling et al. berichteten über komplementäre und alternative Behandlungsmaßnahmen, die meist von den Eltern initiiert werden, um die Lebensqualität zu verbessern [17]. Flankierend zur onkologischen Therapie dient die psychosoziale Betreuung des erkrankten Kindes und seiner Familie, wie sie kürzlich unter Beteiligung der Selbsthilfegruppen in einem umfassenden Aufgabenkatalog zusammengefasst worden ist, der Erleichterung der Akutsituation wie auch der Rehabilitation [40].

Unabhängig von den Funktionen bei der Nachsorge sind die klinischen diagnosebezogenen Tumorregister auch Kompetenzzentren der Therapieplanung und leisten wichtige Beratungen bei der Akutbehandlung von Patienten zum Zeitpunkt der Erstdiagnose, im Therapieverlauf oder in der Rezidivsituation. Diese Konsiliartätigkeit führt nicht nur zu einer individualisierten Therapie im Rahmen des jeweils aktuellen Therapieoptimie-

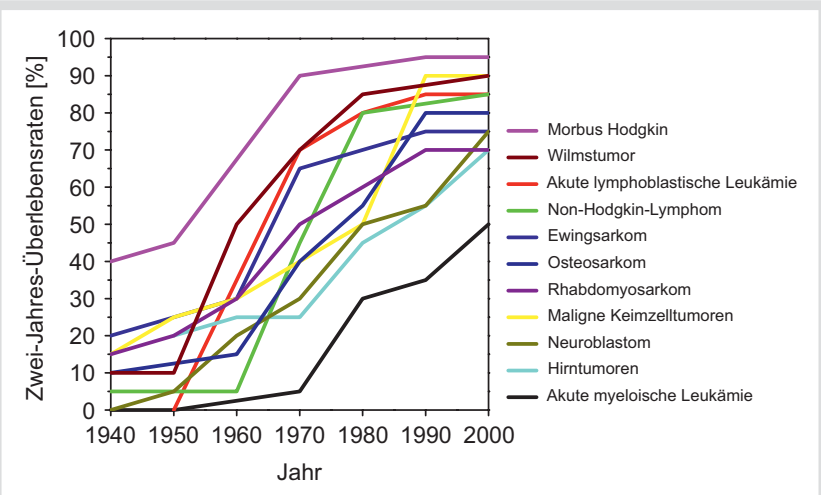

Abb. 1 Zwei-Jahres-Überlebensraten von krebskranken Kindern und Jugendlichen [12, 15]. Gedruckt mit freundlicher Genehmigung des Deutschen Ärzteblattes.

rungsprotokolls sondern erhöht auch die Therapiesicherheit. Die mit dieser Konsiliartätigkeit verbundene Arbeitsbelastung haben Zils et al. für die Krankheitsentität Nephroblastom ermittelt (siehe Seiten 183-188) und gleichzeitig den Benefit dieser Konsultationen für die Patienten durch ein signifikant verbessertes Überleben dokumentiert [45].

\section{Und wo ist das Dilemma?}

$\nabla$

Diese Kurzdarstellung der verschiedenen Arten von Registern im Kompetenznetz der Pädiatrischen Onkologie und Hämatologie macht die unterschiedlichen Arbeitsweisen und Zielsetzungen erkennbar. Weiterhin werden die Synergieeffekte durch Kooperation erkennbar, die dazu geführt haben, dass heute drei von vier Kindern mit der Diagnose Krebs langfristig überleben und bei diesen ganz überwiegend von einer Heilung der Erkrankung auszugehen ist $[12,15]$. Dieser überragende Erfolg ist in vielen kleinen Einzelschritten erreicht worden ( $\bullet$ Abb. 1). Durch die Tätigkeit der klinischen diagnosebezogenen Register gelingt es auch bei sehr seltenen Erkrankungen, bei denen aufgrund der kleinen Fallzahl die Durchführung von randomisierten Studien nicht möglich ist, gesicherte Therapiefortschritte $\mathrm{zu}$ machen [42].

Wie mühsam sich der Erkenntnisgewinn gestalten kann, wird am Beispiel der Mitteilung von Huck et al. (siehe Seiten 147-152) erkennbar, in dem über das sehr gute Langzeitüberleben von sechs konsekutiv mit Knochenmark von HLA-identischen Familienspendern behandelten Patienten mit Fanconi-Anämie berichtet wird [24]. Bei dieser autosomal und/oder X-chromosomal rezessiv vererbbaren Erkrankung besteht eine stark erhöhte Chromosomenbrüchigkeit, die als Ursache für das gehäufte Auftreten von malignen Zweiterkrankungen auch, bzw. besonders nach erfolgreicher Knochenmarktransplantation angesehen wird [38]. Aus komplementationsgenetischen Analysen ist inzwischen bekannt, dass mindestens dreizehn verschiedene Gene dieses Krankheitsbild auslösen können und diese eine wichtige Funktion bei der Reparatur von DNA-Doppelstrangbrücken besitzen [37]. Huck et al. vermuten nun aufgrund einer umfangreichen Literaturanalyse [13,38] und der eigenen kleinen Fallserie, dass das Auftreten einer chronischen Spender-gegenEmpfänger-Krankheit maßgeblich für die Manifestation einer malignen Zweiterkrankung ist, bzw. deren Vermeidung das Malignomrisiko nach Transplantation reduziert [24]. Obwohl es 
schon seit über 20 Jahren ein internationales Fanconi-AnämieRegister gibt [32], sind diese Thesen nicht durch größere Gruppen von gleichartig diagnostizierten und auch gleichartig behandelten Patienten zu verifizieren. Durch kooperative Therapieoptimierungsprotokolle und die gemeinsame Analyse der diagnostischen, therapeutischen und posttherapeutischen Daten werden sich auch bei dieser seltenen Patientengruppe die Synergieeffekte realisieren lassen, wie sie für die kinderonkologischen Patienten inzwischen erreicht worden sind.

Die Zielrichtungen und Arbeitsweisen der unterschiedlichen Register erfordern also den essentiellen Willen zur Kooperation und die Festlegung eindeutig definierter Regeln für den Umgang mit klinischen und wissenschaftlichen Daten. Auch sind eine jeweils adäquate räumliche und personelle Ausstattung nicht überall vorhanden [23]. Die Förderung gestaltet sich für die verschiedenen Formen der Register aufgrund der zu erbringenden Leistungen sehr unterschiedlich.

- Die Gesundheit der Bevölkerung und deren Schutz gehören zu den hoheitlichen Aufgaben eines Staates; deshalb werden die Tumorepidemiologie und die epidemiologische Ursachenforschung zunehmend als öffentliche Aufgabe gesehen [25]. Dies zeigt auch die Verabschiedung des Krebsregistergesetzes durch den Deutschen Bundestag und die nachfolgende Umsetzung durch die Landesparlamente.

- Die exakte Diagnosestellung ist Voraussetzung für eine gezielte Therapie bei einem erkrankten Patienten und gehört zum Leistungskatalog einer Krankenkasse. Im Rahmen von Therapieoptimierungsprüfungen ist zusätzlich auch die Referenzbegutachtung von Tumorgewebe oder von Leukämiezellen notwendig, um einerseits die Diagnose in Zweifelsfällen zu bestätigen und andererseits die risikoadaptierte Therapie auf der Grundlage einheitlicher Beurteilungskriterien im Rahmen von Therapieoptimierungsprüfungen sicherzustellen [44]. Allerdings reicht die Zahl der Untersuchungen bei den malignen Erkrankungen des Kindes- und Jugendalters nicht aus, um eine hierauf spezialisierte Referenzeinrichtung auszulasten und zu finanzieren. Oftmals liegen zudem die Auffassungen der Krankenkassen einerseits und der Studienleitungen bzw. behandelnden Ärzte andererseits über die notwendige Diagnostik und ihre Entgeltung weit auseinander.

- Klinische diagnosebezogene Register erbringen Konsultationen, die auf der Grundlage einer über Jahre aufgebauten und kontinuierlich gepflegten Datei erstellt werden und nicht auf dem aktuell abrufbaren Wissen eines gerade kontaktierten Arztes beruhen. Auch wenn die Konsultation einem bestimmten Patienten zugeordnet werden kann, sind der Aufbau und der Unterhalt des Registers unabhängig von einem Patienten zu sehen und können nicht einer einzelnen Krankenkasse angelastet werden.

- Keinen konkreten Patientenbezug hat die Aufstellung evidenzbasierter Nachsorgeempfehlungen, die in erheblichem Umfang Ressourcen schonen und nur von einem klinischen Register für die entsprechende seltene Erkrankung erarbeitet werden können. Üblicherweise werden bei der Antragstellung für ein Therapieoptimierungsprotokoll derartige Nachsorgeempfehlungen zwingend gefordert und als unentgeltliche Vorleistung erbracht. Damit diese Empfehlungen bei seltenen Erkrankungen dem erforderlichen Evidenzgrad und nicht der Exzellenz des Antragstellers entsprechen, bzw. von Einzelschicksalen determiniert sind, ist die prospektive Erfassung der Spätfolgen durch Kooperation der ausgewiesenen Arbeitsgruppen essentiell [33].
- Ohne konkreten Bezug zur Behandlung eines Patienten arbeiten die Register, die sich mit der Ermittlung von Spätfolgen oder der subjektiven Beurteilung der Lebensqualität beschäftigen. Erst die Erkenntnisse, die sich von einer größeren Zahl von behandelten Patienten ableiten, können in die Therapie zukünftiger Patienten einmünden [1,7,9,10,33].

Je mehr das Interesse des Öffentlichen Gesundheitswesens hinter die individuellen Patientenbelange zurücktritt, umso schwieriger gestaltet sich heute die Finanzierung. Wenn in Vergangenheit die Etablierung von neuen Registern als Qualitätsmerkmal anerkannt und in modellhafter Weise durch begutachtete Drittmittel gefördert wurde, so kommt heutzutage die Erwähnung eines zu etablierenden oder fortzuführenden Registers bei der Antragstellung auf Forschungsförderung einem Ausschlusskriterium gleich. Hierfür sind vor allem zwei Gründe verantwortlich:

- Die Zahl der Register nimmt ständig zu.

- Register sind von ihrer Anlage her langlebig.

\section{Lösungsansätze}

$\nabla$

In dieser Situation ist über neue Formen der Kooperationen nachzudenken, da die knappen Ressourcen der kinderonkologischen Behandlungseinrichtungen mit ihrer Struktur und Ausstattung diese Leistungen ohne zusätzliche Unterstützung nicht erbringen können [23]. So ist unter Forschungsaspekten über die elektronische Krankenakte in einer standardisierten Form nachzudenken, wodurch die Belastungen der Doppeldokumentation vermieden werden [41]. Hierbei sind allerdings unter Wahrung des Datenschutzes die Fragen der Anonymisierung und der Reidentifikation zu lösen, um Doppelmeldungen zu erkennen und nachfolgende Informationen richtig zuzuordnen. Auch könnten bestimmte Aufgaben der Biometrie für mehrere Register gebündelt werden, um Synergieeffekte zu nutzen; der jederzeitige Zugang zu den Registerdaten ist für die Studienleitungen allerdings zu garantieren, um die erforderlichen Konsultationen auf Daten gestützt leisten zu können [11].

Zur langfristigen Finanzierung von diagnosebezogenen klinischen Registern, deren Nutzen für die Forschung und die generelle Behandlung von Patienten mit seltenen Erkrankungen unstrittig sind, sind neue Wege der Finanzierung zu finden.

- Als Voraussetzung sind allgemeinverbindliche Qualitätskriterien festzulegen und Zielvorgaben zu machen, unter denen ein Register als förderungswürdig einzustufen ist. Hierbei sind die Seltenheit und die Schwere der zu registrierenden Erkrankung, der Erfassungsgrad der betroffenen Patienten und die Kooperationen zu berücksichtigen. Auch ist festzustellen, welchen Eigenanteil die beherbergende Institution für den Unterhalt des Registers leisten kann.

- Spätestens bei der Folgefinanzierung sind die Kooperationen zwischen einem weiterzufördernden Register und wissenschaftlichen Projekten besonders zu würdigen. Für die Generierung neuer Erkenntnisse ist der Registeranteil zu definieren bzw. zu belegen und im Antragsverfahren auszuweisen.

- Für die individuell für einen Patienten geleisteten Beratungen im Rahmen der medizinischen Behandlung oder der psychosozialen Betreuung wären differenzierte Leistungskataloge zu erstellen und während einer Erprobungsphase zu validieren. Zweckmäßigerweise wäre diese Erprobungsphase durch ein wissenschaftliches Projekt zur Beurteilung der KostenNutzen-Relation zu begleiten. 


\section{Literatur}

1 Beck JD, Winkler K, Niethammer D, Brandis $M$, Hertzberg H, Hardt $K$, Greil J von der, Überall MA, Rossi R, Lamprecht-Dinnesen A, Brämswig J, Kaatsch P, Michaelis J, Meier W, Hausdorf G, Bielack S, Dörr HG. Die Nachsorge der von einer Krebserkrankung geheilten. Kinder und jungen Erwachsenen Erste Empfehlungen der Arbeitsgemeinsachaft Spätfolgen. Klin Pädiatr 1995; 207: 186-192

2 Berthold F, Bode G, Böcker A, Christaras A, Creutzig U, Henze G, Herold $R$, Heyll A, Malzahn J, Rath T, Jürgens $H$. Maßnahmen zur Qualitätssicherung für die stationäre kinderonkologische Versorgung. Klin Pädiatr 2006; 218: 293-295

3 Berthold F, Burdach S, Kremens B, Lampert F, Niethammer D, Riehm H, Ritter J, Treuner J, Utsch S, Zieschang J. The role of chemotherapy in the treatment of chlidren with neuroblastoma stage IV: The GPO (German Pediatric Oncology Society) experience. Klin Pädiatr 1990; 202: 262-269

4 Berthold F, Kaatsch $P$, Evers $G$, Harms $D$, Jürgens $H$, Niethammer $D$, Ritter J, Wahlen W, Treuner J, Lampert F. Intensive Kombinationschemotherapie und $\beta$-Interferon zur Behandlung von Kindern mit metastasiertem Neuroblastom: Studie GPO-NB 79/82. Klin Pädiatr 1984; 196: $143-149$

5 Biskup W, Calaminus G, Schneider DT, Leuschner I, Göbel U. Teratoma with malignant transformation: Experiencies of the cooperative GPOH protocols MAKEI 83/86/89/96. Klin Pädiatr 2006; 218: 303-308

6 Bölling T, Ernst I, Könemann S, Willich N. Pediatric Radiation Oncology in Germany: A study of availability and application. Klin Pädiatr 2008; 220: 178-182

7 Bölling T, Schuck A, Pape H, Rübe C, Meyer FM, Martini C, Timmermann $B$, Asadpour B, Kortmann RD, Beck JD, Langer T, Paulides M, Könemann $S$, Willich $N$. Registry for the evaluation of late side effects after radiotherapy in childhood and adolescence - first results. Klin Pädiatr 2007: 219: 139-145

8 Calaminus G, Kaatsch P. Positionspapier der Gesellschaft für Pädiatrische Onkologie und Hämatologie (GPOH) zu (Langzeit-)Nachbeobachtung, (Langzeit-)Nachsorge und Spätfolgenerhebung bei pädiatrisch-onkologischen Patienten. Klin Pädiatr 2007; 219: 173-178

9 Calaminus G, Weinspach S, Teske C, Göbel U. Quality of life in children and adolescents with cancer, first results of an evaluation of 49 patients with PEDQOL-questionaire. Klin Pädiatr 2000; 212: 211-215

10 Calaminus, Weinspach S, Teske C, Göbel U. Überlebensqualität von Kindern und Jugendlichen nach Krebserkrankung: Einfluss der Spätfolgen auf die gesundheitsbezogene Lebensqualität. Klin Padiatr 2007; 219: $173-178$

11 Creutzig U, Hannemann J, Krämer I, Zimmermann M, Herold R, Marx JF. Das Qualitätshaus als Instrument zur Leistungsverbesserung von Studienzentralen der Pädiatrischen Onkologie und Hämatologie. Klin Pädiatr 2005; 217: 114-119

12 Creutzig U, Henze G, Bielack S, Herold R, Kaatsch P, Klussmann JH, Graf $N$, Reinhardt $D$, Schrappe $M$, Zimmermann $M$, Jürgens $H$. Krebserkrankungen im Kindesalter. Erfolg durch einheitliche Therapiekonzepte seit 25 Jahren. Deutsches Ärzteblatt 2003; 100: A842-A852

13 Farzin A, Davies SM, Smith FO, Filipovich A, Hansen M, Auerbach AD, Harris RE. Matched sibling donor haematopoietic stem cell transplantation in Fanconi anaemia: an update of the Cincinnati Children's experience. Br J Haematol 2007; 136: 633-640

14 Fischer M, Spitz R, Oberthür A, Westermann F, Berthold F. Risk estimation of neuroblastoma patients using molecular markers. Klin Pädiatr 2008; 220: 137-146

15 Göbel U, Fischer R, Henze G. Von der Therapiestudie zur Qualitätssicherung. Klin Pädiatr 1997; 209: 145-146

16 Göbel U, Calaminus G, Schneider DT, Bernbeck B, Koch S, Harms D. The malignant potential of teratomas. The MAKEI experiences and implications for the new protocol. Klin Pädiatr 2006; 218: 309-314

17 Gottschling S, Längler A, Tautz C, Graf N. Complementary and alternative medicine in pediatric oncology. Klin Pädiatr 2006; 218: 157-164

18 Harbott J. Chromosomenabberationen bei akuten Leukämien im Kindesalter. Enke, Stuttgart 1993

19 Harms D. Kieler Kindertumorregister. Dt Ärztebl 1996; 93: A746A754

20 Harms D, Lennert K. Zur Frage des zentralen Tumorregisters der Gesellschaft. Klin Pädiatr 1978; 190: 5-8

21 Harms D, Zahn S, Göbel U, Schneider DT. Pathology and molecular biology of teratomas in childhood and adolescence. Klin Pädiatr 2006; 218: 296-302

22 Hero B, Simon T, Spitz R, Ernestus K, Gnekow AK, Scheel-Walter HG, Schwabe D, Schilling FH, Benz-Bohm G, Berthold F. Localized infant neuroblastoma may regularly show spontaneous regression: Results of the prospective trials NB95-S and NB97. J Clin Oncol in press
23 Herold $R$, Reiche R, Creutzig U, Henze G. Stand und Bewertung der Strukturen und Ausstattung pädiatrisch-onkologisch-hämatologischer Kliniken. Klin Pädiatr 2007; 219: 380-390

24 Huck K, Hanenberg H, Nürnberg W, Dilloo D, Burdach S, Göbel U, Laws $H J$. Favourable long-term outcome after matched sibling transplantation for Fanconi anemia (FA) and in vivo T-cell depletion. Klin Pädiatr 2008; 220: 147-152

25 Kaatsch $P$. Das Deutsche Kinderkrebsregister im Umfeld günstiger Rahmenbedingungen. Bundesgesundheitsblatt Gesundheitsforschung Gesundheitsschutz 2004; 47: 437-443

26 Kaatsch P, Blettner M, Spix C, Jürgens H. Das Langzeit-Follow-up in der deutschen pädiatrischen Onkologie als Basis für die Durchführung von Studien mit Langzeitüberlebenden. Klin Pädiatr 2005; 217: 169-175

27 Kaatsch P, Kaletsch U, Krummenauer F, Meinert R, Miesner A, Haaf G, Michaelis J. Fallkontrollstudie zu den Ursachen von Leukämien bei Kindern in Niedersachsen - Durchführung und Ergebniszusammenstellung. Klin Pädiatr 1996; 208: 179-185

28 Kaatsch P, Michaelis J. Das kooperative Dokumentationsprojekt für kindliche Malignome, Klin. Pädiatr 1981; 193: 157-263

29 Kaatsch P, Spix C. Jahresbericht 2006/2007 (1980-2006) - Deutsches Kinderkrebsregister, http://www.kinderkrebsregister.de/

30 Kaatsch P, Spix C, Schulze-Rath R, Schmiedel S, Blettner M. Leukaemia in young children living in the vicinity of German nuclear power plants. Int J Cancer 2008; 122: 721-726

31 Kaatsch P, Steliarova-Foucher E, Crocetti E, Magnani C, Spix C, Zambon $P$. Time trends of cancer incidence in European children (1978-1997): report from the ACCIS project. Eur J Cancer 2006; 42: 1961-1971

32 Kutler DI, Singh B, Satagopan J, Batish SD, Berwick M, Giampietro PF, Hanenberg $H$, Auerbach AD. A 20-year perspective on the International Fanconi Anemia Registry (IFAR). Blood 2003; 101: 1249-1256

33 Langer T, Stöhr W, Paulides W, Kremers A, Dörr H-G, Göbel U, Beck DJ. Prospective multicenter registration of major late sequelae in sarcoma patients using the late effects surveillance system (LESS). Klin Pädiatr 2005; 217: 176-181

34 Ludwig WD, Harbortt J, Bartram CR, Komischke B, Sperling C, Teichmann $J V$, Seibt-Jung $H$, Notter $M$, Odenwald $E$, Nehmer A et al. Incidence and prognostic significance of immunophenotyppic subgroups in childhood acute lymphoblastic leukemia: experience of the BFM study 86. Recent Results Canc Res 1993; 131: 269-282

35 Michaelis J, Haaf G, Zöllner J, Kaatsch P, Krummenauer F, Berthold F. Fallkontrollstudie zum Anstieg der Neuroblastominzidenz in Deutschland nach dem Tschernobyl-Unfall. Klein Pädiatr 1996; 208: 172178

36 Möricke A, Zimmermann M, Reiter A, Gadner H, Odenwald E, Harbott J, Ludwig WD, Riehm H, Schrappe M. Prognostic impact of age in children and adolescents with acute lymphoblastic leukemia: data from the trials ALL-BFM 86, 90, and 95. Klin Pädiatr 2005; 217: 310-320

37 Niedernhofer LJ, Lalai AS, Hoeijmakers JH. Fanconi Anemia (cross-)linked to DNA repair. Cell 2005; 123: 1191-1198

38 Rosenberg PS, Socie G, Alter BP, Gluckman E. Risk of head and neck squamous cell cancer and death in patients with Fanconi anemia who did and did not receive transplants. Blood 2005; 105: 67-73

39 Schilling FH, Spix C, Berthold F, Erttmann R, Fehse N, Hero B, Klein G Sander J, Schwarz K, Treuner J, Zorn U, Michaelis J. Neuroblastoma screening at one year of age. N Engl J Med 2002; 346: 1047-1053

40 Schreiber-Gollwitzer B, Gallo A Di, Maier S. Position paper by the Gesellschaft für Pädiatrische Onkologie und Hämatologie $(\mathrm{GPOH})$ and the Deutsche Leukämie-Forschungshilfe (DLFH) on psycho-social care for pediatric oncology and haematology patients in hospital. Klin Pädiatr 2007; 219: 368-371

41 Telematikplattform für Medizinische Forschungsnetze e.V., http:// www.tmf-ev.de/

42 Thiele KP, Rheineberger P. „Unmöglich“ gibt es nicht. Deutsches Ärzteblatt 2003; 100: A1044-A1046

43 Walter KN, Kratz C, Uhl M, Niemeyer C. Chemotherapy as a therapeutic option for congenital neuroblastoma complicated by paraplegia. Klin Pädiatr 2008; 220: 175-177

44 Weißbach L, Fischer R, Bredenkamp R, Göbel U, Hart D, Hermanek $P$, Hiddemann $W$, Jonat $W$, Rossion I, Sindermann H. Therapieoptimierungsprüfung. Eine Notwendigkeit für die Versorgung krebskranker Patienten. Onkologe 1999; 5: 1015-1020

45 Zils K, Furtwängler R, Reinhard H, Alkassar M, Graf N. Consultation within the nephroblastoma trial SIOP 2001/GPOH as part of the workload in the trial office. Klin Pädiatr 2008; 220: 183-188 